\title{
Bases para el desarrollo de un modelo de rehabilitación forestal en minería utilizando Nothofagus pumilio [Poepp.et Endl] Krasser
}

\author{
Bases to develop a coal mine reclamation model using \\ Nothofagus pumilio [Poepp.et Endl] Krasser
}

Patricio Valenzuela ${ }^{1}$, Eduardo Arellano ${ }^{1,2}$, James Burger ${ }^{3}$,

Gabriel Zegers ${ }^{1} \&$ Ignacio Fernández ${ }^{4}$

Búsqueda de un modelo para

rehabilitación de sitios perturbados

por minería en la Patagonia Occidental

Desde inicios de la colonización en Magallanes y la Patagonia occidental, el paisaje ha sido objeto de un proceso continuo de cambios y transformaciones en sus patrones ecológicos (Martinic, 2004; Otero, 2006). Los ecosistemas forestales del tipo Nothofagus pumilio y en asociaciones con Nothofagus betuloides y Nothofagus antarctica, han sido testigos de un importante proceso de degradación como consecuencia del aprovechamiento forestal y establecimiento de praderas de uso ganadero (Huber \& Markgraf, 2003).

Extensiones entre 200.000 y 300.000 ha (Cruz \& Lara, 1987; Promis, 2008), fueron taladas y quemadas para su uso ganadero ovino y para la minería del carbón desde el comienzo de la colonización en Magallanes. Estas primeras explotaciones carboniferas se realizaron en Brunswick, en la costa del mar de Skyring y en la Isla Riesco (Mina Helena) hasta la década de 1950 (Martinic et al., 2011). Posteriormente y a mayor escala, los pasivos mineros, como el dejado por Mina Pecket en la península de Brunswick (Martinic, 2004), son hoy en día un ejemplo de manejo donde no se consideró las condiciones bióticas y abióticas que promovieran el establecimiento de especies en el largo plazo. Tarea que ha demostrado ser viable en este tipo de pasivos mineros con las consideraciones adecuadas (Felinks \& Wiegleb, 1999; Shulz \& Wiegleb, 2000).

A nivel mundial se implementan diversas for- mas de rehabilitación en los pasivos o botaderos mineros. La actividad más común es la reposición de suelo superficial y posterior siembra de especies herbáceas invasivas, las cuales llegan a un rápido aumento de cobertura y reducen los costos en trabajo que implican alternativas de rehabilitación más complejas (Parrotta \& Knowles ,1999; Camargo et al., 2002). No obstante esta técnica presenta limitantes en el establecimiento de especies nativas debido a la gran compactación por el uso de maquinaria, altos costos de mantención y la competencia con herbáceas exóticas (Ashby, 1991; Unger \& Cassel, 1991; Skousen et al. 2011). Además de estas limitaciones se ha buscado integrar los pasivos mineros con el entorno forestal, profundizado en la generación de modelos alternativos de rehabilitación que busquen aumentar la calidad de los sustratos y la compatibilidad entre las especies (Tongway \& Ludwig, 2011).

\footnotetext{
1 Departamento de Ecosistemas y Medioambiente, Pontificia Universidad Católica de Chile.

valenzuelac.patricio@gmail.com $>$

gabrielzegersm@gmail.com

2 Center of Applied Ecology and Sustainability (Capes),

Pontificia Universidad Católica de Chile.

eduardoarellano@uc.cl

3 Department of Forest Resources and Environmental Conservation, Virginia Tech, Blackbusrg, VA, USA. jrburger@vt.edu

4 Landscape Ecology \& Sustainability Laboratory, Arizona State University, Tempe, AZ. ifernandech@gmail.com
} 


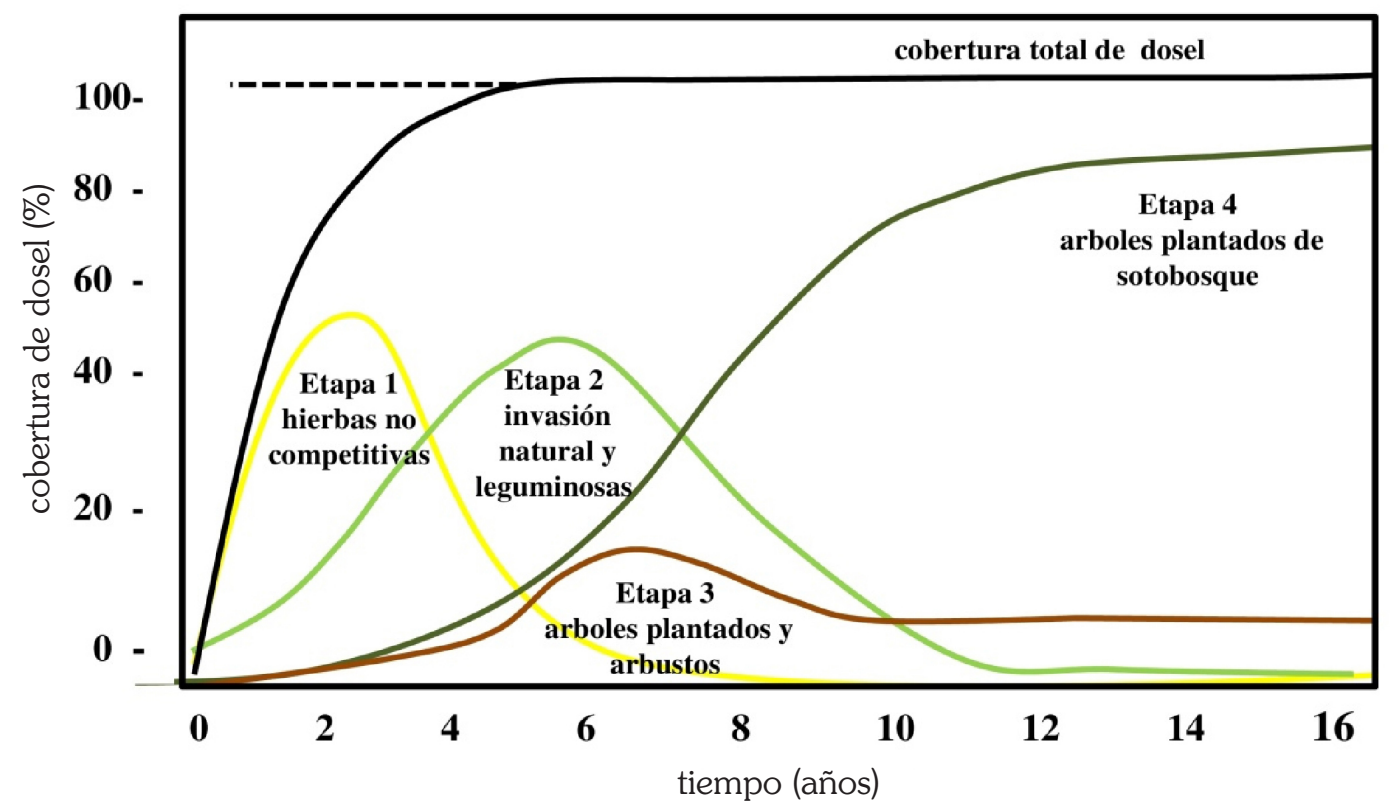

Fig 1. Forestry Reclamation Approach, "FRA", busca acelerar el proceso natural de sucesión. Todos los tipos vegetacionales son establecidos durante la rehabilitación. Con el paso del tiempo, los rendimientos de herbáceas y leguminosas apoyan el crecimiento de árboles nativos y el posterior aumento de árboles comerciales, cuando es posible (Burger \& Zipper, 2011).

Un modelo de rehabilitación donde se incorporan elementos de un sistema antes de la intervención minera es el llamado modelo de aproximación forestal a la rehabilitación (Forest Reclamation Approach, FRA). Este nació al alero de la minería del carbón en los Apalaches y se ha aplicado en la recuperación de miles de pasivos mineros producidos por la extracción de carbón a cielo abierto (Zipper et al., 1989; Torbert \& Burger, 2000).

En el desarrollo de FRA se busca recuperar el suelo superficial, las especies arbustivas $y$ arbóreas, las cuales se integran al sitio acelerando la sucesión para estabilizar el terreno y controlar la erosión, teniendo en cuenta una relación costo-efectiva (Burger et al. 2005; Sweigard et al. 2007). Se establecen casi simultáneamente una combinación de herbáceas, leguminosas (usadas cuando sea necesario), arbustos nodrizas y árboles, esperando que cada tipo de planta cumpla una función específica y aumente el éxito de las siguientes especies, correspondientes a un estado sucesional posterior (Fig. 1) (Burger \& Zipper, 2011). La secuencia de pasos para alcanzar esta recuperación es la siguientes: 1 . Crear un medio con una profundidad mayor a 1 metro, que sea adecuado para que se desarrollen las raíces de los árboles. Se utiliza como cobertura el suelo superficial (con materia vegetal) y algún pasivo minero adecuado, extraído de la actividad minera. 2. Usar este material recuperado y aplicarlo sin compactar en los sitios donde se plantará con herbáceas, arbustos y árboles. 3. Uso de coberturas que deben ser compatibles con el crecimiento de los árboles. 4. Plantación de árboles protectores y arboles comerciales. 5. Usar técnicas de plantación considerando las necesidades de cada especie.

El objetivo de este trabajo fue caracterizar variables del suelo y de la vegetación que puedan resultar útiles para al momento de adaptar el modelo FRA en sitios donde se desarrollara un proyecto minero y en los cuales alguna vez hubo bosques mixtos de Nothofagus spp. en la Isla Riesco. Mediante descripción de cobertura vegetacional, especies predominantes y micrositios facilitadores de la regeneración, buscamos obtener información para optimizar futuras tareas de rehabilitación en sitios sometidos a actividades de minería de carbón a cielo abierto. 
Recopilación de antecedentes en un área de estudio intervenida por minería

En terrenos de la Estancia Invierno (coordenadas UTM 19F, 324614.E; 4137080.S), propiedad de Mina Invierno, S.A., Comuna de Río Verde, Provincia de Magallanes, XII Región de Magallanes y de la Antártica Chilena fue el sitio escogido dado el inminente impacto por la extracción de carbón en la zona. Los tipos vegetacionales en esta Isla corresponden a las Regiones Vegetacionales de: (1) Matorral y Estepa Patagónica, Subregión de la Estepa Patagónica de Magallanes, y (2) Región del Bosque Andino patagónico, Subregión de las Cordilleras Patagónicas (Gajardo, 1994).

Las actividades de terreno se realizaron en el verano del año 2011, previo al inicio de las actividades de explotación minera. El área de impacto corresponde a 1.346 ha donde se definieron 4 ecotipos de vegetación representativos en función de la estructura de la vegetación dominante: (1) 232 ha Bosque de Nothofagus pumilio y Nothofagus betuloides, perturbados con impacto silvopastoril (BP), (2) Matorral de transición bosque-pradera dominado por Berberis microphylla (MT), (3) Matorral higrófilo asociado a vegas, dominado por Nothofagus antarctica (MH) y (4) Praderas ganaderas dominadas por especies invasivas (PG). Estos tres últimos sectores tienen una extensión de 1.106 ha.

En cada uno de los ecotipos identificados se realizaron 12 parcelas de muestreo de $4 \mathrm{x}$ 25 metros. Para determinar este número de parcelas se utilizó una curva de saturación, de manera que las parcelas fuesen representativas vegetacionalmente. En cada parcela se registró la composición florística y la cobertura porcentual de las especies más abundantes y representativas de sitios donde interactúan especies nativas con herbáceas invasivas (Domínguez, 2010). Dentro de cada parcela se establecieron 10 cuadrantes de $0,25 \mathrm{~m}^{2}$, dispuestos cada 5 metros en los bordes más largos de las parcelas (5 cuadrantes por lado, incluyendo las cuatro esquinas). Se cuantificó la frecuencia relativa de las especies presentes y el porcentaje total de ellas en relación a su presencia en los cuadrantes.

Para evaluar la regeneración de plántulas correspondientes a especies del genero Nothofagus spp. consideramos las unidades de crecimiento (GU) (Puntieri et al., 1999) en individuos con alturas menores a $30 \mathrm{~cm}$, también se consideró aquellas plántulas con tamaño menor a $150 \mathrm{~cm}$ de altura y $3 \mathrm{~cm}$ de diámetro en los cuadrantes de $0,25 \mathrm{~m}^{2}$. Con el número de plántulas se calculó el promedio de regeneración para cada especie por ecotipo correspondiente. Para especies arbustivas se tuvo en cuenta la presencia de plántulas con tamaño menor $20 \mathrm{~cm}$ y rebrotes de plantas presentes.

Para el efecto de las características de micrositio sobre la regeneración arbórea se establecieron 15 parcelas de $0,25 \mathrm{~m}^{2}$ en sectores contiguos a cada una de las parcelas utilizadas para evaluar la composición. 5 cuadrantes fueron ubicados contiguos a troncos, 5 bajo los arbustos y los 5 restantes en sectores abiertos. La regeneración fue medida con la misma metodología mencionada anteriormente. Para comparar diferencias entre los micrositios se realizó un análisis $\operatorname{Glm}(p<0,05)$.

La característica de los suelos presentes en el área de estudio se determinó mediante la construcción de calicatas de 2,2 metros de profundidad promedio. En total se realizaron 33 calicatas las cuales fueron distribuidas acorde a las muestras representativas de los 4 ecotipos presentes en la zona de estudio. Los suelos se caracterizaron por color, textura, plasticidad, estructura, profundidad de cada horizonte y características propias de cada perfil. Se tomaron 3 muestras de suelo en cada calicata, considerando los primeros horizontes de cada perfil, luego las muestras fueron sujetas a análisis nutricional y de fertilidad en el laboratorio de Servicios ubicado en la Facultad de Agronomía e Ingeniería Forestal de la Pontificia Universidad Católica de Chile. Con los datos obtenidos se realizó un mapa de zonificación de las distintas comunidades y tipos de suelos presentes en el área, para lo cual se utilizó ArcMap 9.3.

\section{Caracterización vegetacional}

Los resultados obtenidos en la zona de muestreo indican que las principales especies presentes corresponden a los 4 ecotipos seleccionados (Fig. 2). En el bosque perturbado predominó Nothofagus pumulio y Nothofagus 
Tabla 1. Listado de especies principales en las conformaciones vegetacionales donde se realizó el muestreo.

\begin{tabular}{|c|c|c|}
\hline $\begin{array}{l}\text { Bosque perturbado con } \\
\text { impacto silvopastoril (PB) }\end{array}$ & Cobertura (\%) & Frecuencia (\%) \\
\hline Nothofagus pumilio & 48 & 41 \\
\hline Nothofagus betuloide & 20 & 21 \\
\hline Acaena spp. & 11 & 21 \\
\hline Berberis ilicifolia & 4 & 15 \\
\hline Holcus lanatus & 4 & 17 \\
\hline Gaultheria pumilia & 4 & 5 \\
\hline Matorral de transición (MT) & Cobertura (\%) & Frecuencia (\%) \\
\hline Berberis microphylla & 36 & 72 \\
\hline Cotula scariosa & 11 & 33 \\
\hline Trifolium repens & 7 & 51 \\
\hline Gunnera magellanica & 7 & 27 \\
\hline Blechnum penna-marina & 6 & 21 \\
\hline Taraxacum officinalis & 6 & 67 \\
\hline Pradera ganadera (PG) & Cobertura (\%) & Frecuencia (\%) \\
\hline Festuca spp. & 17 & 42 \\
\hline Holcus lanatus & 17 & 80 \\
\hline Trifolium repens & 12 & 64 \\
\hline Berberis microphylla & 10 & 31 \\
\hline Gaultheria pumilia & 9 & 22 \\
\hline Acaena pinnatifida & 9 & 62 \\
\hline Matorral Higrófilo (MH) & Cobertura (\%) & Frecuencia (\%) \\
\hline Gunnera magellanica & 26 & 56 \\
\hline Festuca spp. & 24 & 56 \\
\hline Holcus lanatus & 22 & 92 \\
\hline Nothofagus antarctica & 18 & 22 \\
\hline Cotula scariosa & 15 & 36 \\
\hline Poa nemoralis & 15 & 36 \\
\hline
\end{tabular}

betuloides, lo que cambia fuertemente en otros sitios, donde predominaron especies arbustivas como Berberis microphylla y Cotula scariosa en Matorral de Transición (MT) y herbáceas invasoras como Holcus lanatus en sitios de Pradera (PG).

Los géneros más representados fueron Notofagus, Berberis, Poa y Ranunculus, cada uno de ellos con tres especies. De acuerdo con el ecotipo, la mayor diversidad la presento MT con 43 especies, seguido por el MH, BP y PG, con 33, 32 y 30 especies, respectivamente. El mayor ecotipo con especies introducidas es la PG, con 9 especies que representan el 29\% del total de las especies reconocidas en esa comunidad. 

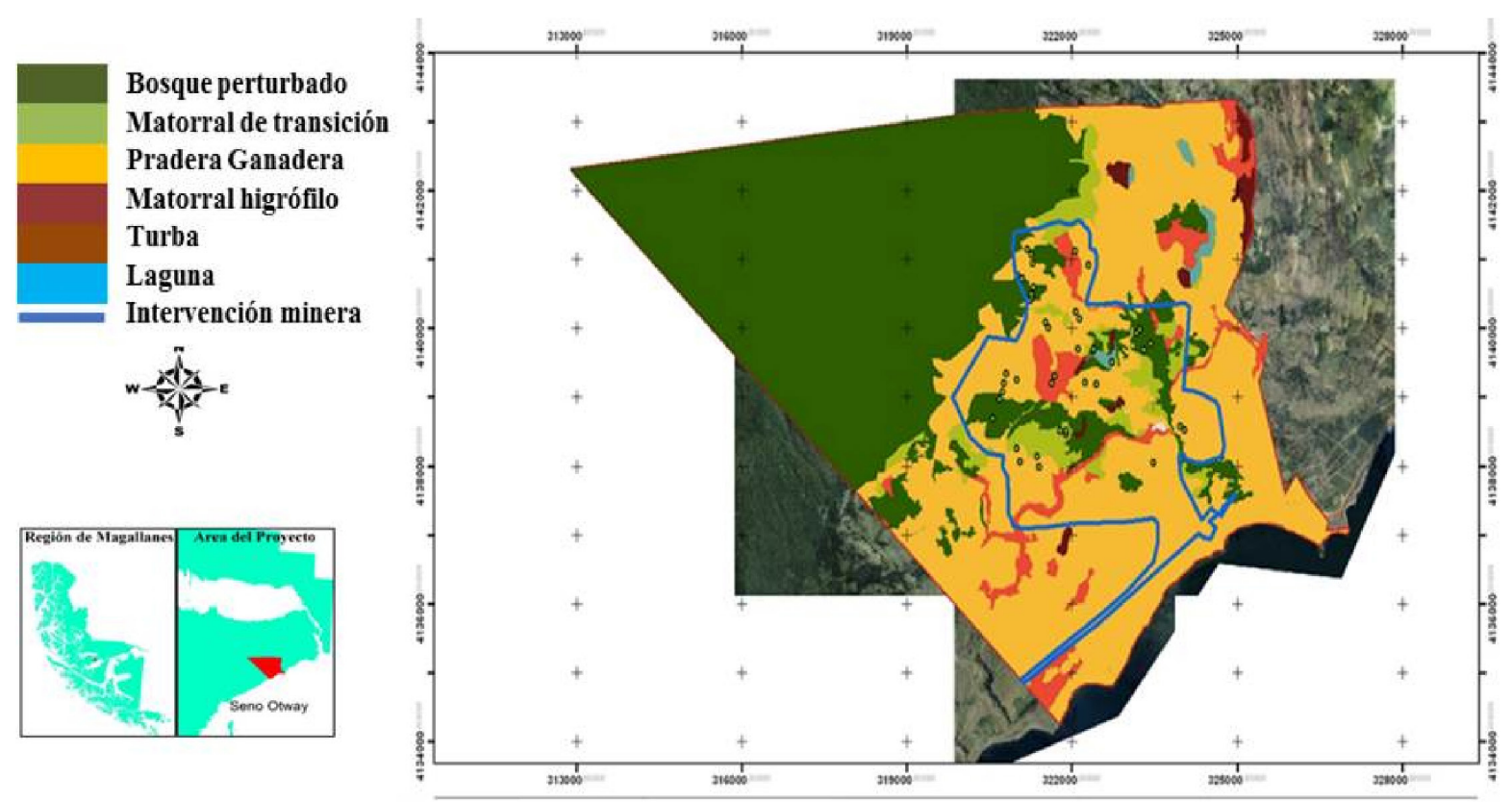

Fig 2. Distribución de las principales comunidades y ecotipos definidos en la caracterización vegetacional. El borde coloreado de azul indica el área de intervención minera.

Patrones de cobertura

En BP las especies de mayor cobertura correspondieron a dos especies, Nothofagus pumilio y Notothofagus betuloides, las cuales representan cerca del $70 \%$ de la cobertura de la comunidad. En el sotobosque es posible apreciar arbustos propios de la comunidad como Berberis ilicifolia y Gaultheria pumila, con 4,2\% y 4,0\% de la cobertura total.

En MT la especie predominante fue el arbusto Berberis microphylla con un 36,5 de cobertura, la cual apareció acompañada herbáceas como Trifolium repens y Gunnera magellanica, que en su conjunto suman un $25 \%$ de cobertura. Se destaca la presencia del helecho nativo Blechnum penna-marina con un $6,1 \%$ de cobertura (Tabla 1). En PG predominan las coberturas de especies herbáceas invasivas y naturalizadas, junto a algunas nativas, como Festuca sp., las cuales suman un 90\% de cobertura, destacando entre las especies Holcus lanatus, Festuca sp. y Trifolium repens con $16,6 \%, 16,7$ y $12,1 \%$ de la cobertura. En Matorral Higrófilo destaca la presencia de Gunnera magellanica con $26 \%$ de cobertura, junto a ella se presentan Festuca sp. (24,4\%), Holcus lanatus
$(22,4)$ y Cotula scariosa $(14,6)$ (Tabla 1$)$.

Estrategias de regeneración natural

De todas las especies leñosas nativas encontradas en el área de estudio, solo 5 mostraron evidencias de estar regenerando en los sitios de muestreo. La especie que presenta las mejores respuestas es Berberis microphylla, seguida por Nothofagus pumilio en BP. Es importante mencionar las diferencias entre los ecotipos definidos dado que en BP, Nothofagus spp. predomina fuertemente, sin embargo la respuesta es totalmente distinta en los otros ecotipos como MT donde es posible observar altas tasas de regeneración para Berberis microphylla (Tabla 2). En PG la regeneración es baja para especies arbóreas y arbustivas, solo Berberis microphylla muestra la capacidad de recolonizar en algunos sitios dominados por especies ganaderas.

Facilitación de micrositios para especies arbóreas

En BP aparece una mayor regeneración bajo troncos (muertos horizontal), seguido por sitios 
Tabla 2. Tasas de regeneración vegetacional donde se indican los valores promedios de plántulas \pm error estándar por parcela. El listado solo incorpora las especies leñosas categorizadas por ambiente. En la tabla s/r significa sin regeneración.

\begin{tabular}{|c|c|c|c|c|}
\hline Especie & Bosque & M. Transición & M. Higrófilo & Pradera \\
\hline Berberis darwinii & $\mathrm{s} / \mathrm{r}$ & $\mathrm{s} / \mathrm{r}$ & $\mathrm{s} / \mathrm{r}$ & $\mathrm{s} / \mathrm{r}$ \\
\hline Berberis ilicifolia & $0,90 \pm 0,50$ & $\mathrm{~s} / \mathrm{r}$ & $\mathrm{s} / \mathrm{r}$ & $\mathrm{s} / \mathrm{r}$ \\
\hline Berberis microphylla & $1,50 \pm 1,39$ & $9,47 \pm 3,15$ & $3,80 \pm 2,11$ & $2,05 \pm 0,98$ \\
\hline Empetrum rubrum & $s / r$ & $\mathrm{~s} / \mathrm{r}$ & $\mathrm{s} / \mathrm{r}$ & $\mathrm{s} / \mathrm{r}$ \\
\hline Gaultheria mucronata & $\mathrm{s} / \mathrm{r}$ & $\mathrm{s} / \mathrm{r}$ & $\mathrm{s} / \mathrm{r}$ & $\mathrm{s} / \mathrm{r}$ \\
\hline Gaultheria pumilia & $\mathrm{s} / \mathrm{r}$ & $0,18 \pm 0,17$ & $3,80 \pm 3,80$ & $0,05 \pm 0,05$ \\
\hline Maytenus magellanica & $\mathrm{s} / \mathrm{r}$ & $\mathrm{s} / \mathrm{r}$ & $\mathrm{s} / \mathrm{r}$ & $s / r$ \\
\hline Nothofagus antarctica & $\mathrm{s} / \mathrm{r}$ & $\mathrm{s} / \mathrm{r}$ & $\mathrm{s} / \mathrm{r}$ & $\mathrm{s} / \mathrm{r}$ \\
\hline Nothofagus betuloide & $0,20 \pm 0,20$ & $0,06 \pm 0,08$ & $\mathrm{~s} / \mathrm{r}$ & $0,09 \pm 0,06$ \\
\hline Nothofagus pumilio & $2,00 \pm 1,58$ & $1,18 \pm 0,72$ & $0,20 \pm 0,20$ & $0,09 \pm 0,06$ \\
\hline
\end{tabular}
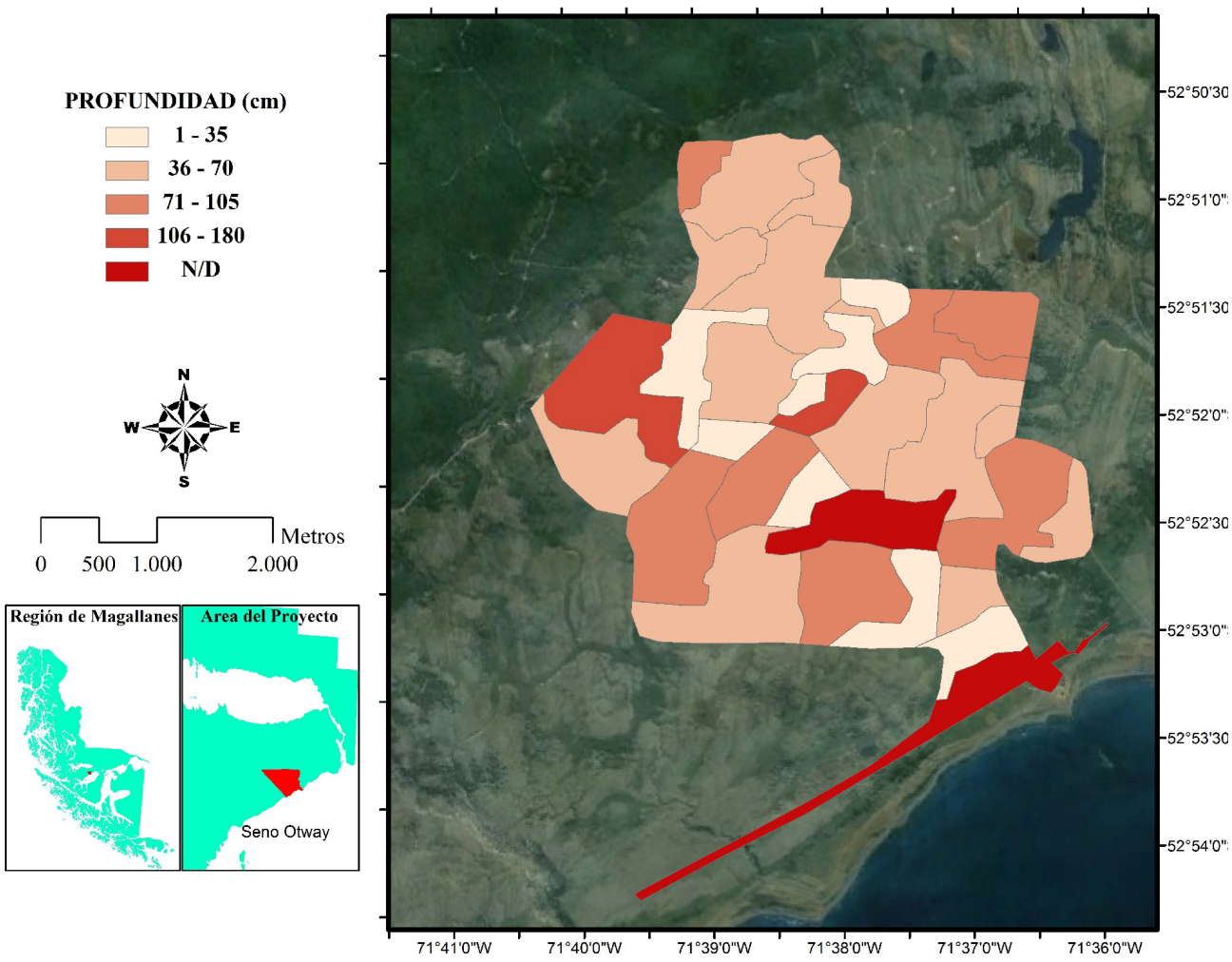

Fig. 3. Distribución de las profundidades en estratas de suelo mineral encontrado en el área de intervención minera, este registro se realizó en los mismos lugares donde se definieron las principales comunidades y ecotipos durante la caracterización vegetacional. 
Tabla 3. Tasas de regeneración vegetacional promedio \pm error estándar en cada ecotipo por micrositio correspondiente. El signo $\left(^{*}\right)$ indica Significancia con $\mathrm{p}<0,05$ en prueba de Chi cuadrado $\left(\mathrm{X}^{2}\right)$.

\begin{tabular}{|c|c|c|c|c|}
\hline \multirow[t]{2}{*}{ Comunidad } & \multirow[t]{2}{*}{ Micrositios } & \multicolumn{3}{|c|}{ Especies } \\
\hline & & N. pumilio & N. betuloides & N. antarctica \\
\hline Bosque Perturbado & Abierto & $1,20 \pm 0,55$ & $1,0 \pm 0,10$ & $\mathrm{~s} / \mathrm{r}$ \\
\hline Bosque Perturbado & Arbusto & $1,60 \pm 0,60$ & $0,30 \pm 0,21$ & $\mathrm{~s} / \mathrm{r}$ \\
\hline Bosque Perturbado & Tronco & $1,70 \pm 0,70$ & $1,00 \pm 0,39^{*}$ & $\mathrm{~s} / \mathrm{r}$ \\
\hline Matorral de Transición & Abierto & $1,35 \pm 0,31^{*}$ & $0,24 \pm 0,14$ & $\mathrm{~s} / \mathrm{r}$ \\
\hline Matorral de Transición & Arbusto & $2,77 \pm 0,38$ & $0,77 \pm 0,24$ & $\mathrm{~s} / \mathrm{r}$ \\
\hline Matorral de Transición & Tronco & $3,00 \pm 0,42$ & $\mathrm{~s} / \mathrm{r}$ & $1,80 \pm 0,49$ \\
\hline Matorral Higrófilo & Abierto & $1,00 \pm 0,45$ & $\mathrm{~s} / \mathrm{r}$ & $1,20 \pm 0,80$ \\
\hline Matorral Higrófilo & Arbusto & $2,00 \pm 0,84$ & $\mathrm{~s} / \mathrm{r}$ & $1,20 \pm 0,80$ \\
\hline Matorral Higrófilo & Tronco & $1,40 \pm 0,75$ & $\mathrm{~s} / \mathrm{r}$ & $0,60 \pm 0,40$ \\
\hline Pradera ganadera & Abierto & $1,14 \pm 0,10^{*}$ & $1,20 \pm 0,55$ & $\mathrm{~s} / \mathrm{r}$ \\
\hline Pradera ganadera & Arbusto & $1,55 \pm 0,17$ & $1,20 \pm 0,55$ & $\mathrm{~s} / \mathrm{r}$ \\
\hline Pradera ganadera & Tronco & $1,64 \pm 0,19$ & $1,20 \pm 0,55$ & $s / r$ \\
\hline
\end{tabular}

donde se presentan arbustos nodriza. Para Nothofagus betuloides es posible observar diferencias significativas entre la regeneración cercana a troncos en comparación a otro tipo de micrositio (Tabla 3). En MT y PG, Nothofagus pumilio muestra una mayor respuesta en regeneración en micrositios de troncos y nodrizas protectoras. En Matorral Higrófilo no existe evidencia de un efecto facilitador dado por arbustos u otra protección (Tabla 3).

Caracterización de suelos para selección de sustratos superficiales

Existe una alta variabilidad espacial en las propiedades de suelo, no obstante fue posible apreciar un patrón general de textura franca-arenosa a franca en la estrata superficial. Bajo esta capa aparece un sustrato de origen fluvioglacial que en general proporciona un drenaje adecuado. Sin embargo, en posiciones más drepresional del terreno es posible observar situaciones de anegamiento estacional a profundidades de 1 metro o más, lo que otorga al suelo una coloración gris.

La profundidad del horizonte mineral, con materia orgánica humificada, variaron de $30 \mathrm{~cm}$ en algunos sitios a bajo los $180 \mathrm{~cm}$ en otros, tal como se puede observar en la Fig. 2. El horizonte mineral superficial corresponde a la estrata que contiene la materia orgánica humificada o estabilizada que normalmente se encuentra sobre el suelo mineral y es de alta importancia en las propiedades biológicas y ecológicas de éste. Al comparar la profundidad de suelo y las comunidades vegetacionales, se pudo establecer que la comunidad vegetacional de pradera $(\mathrm{PG})$ tuvo un horizonte $\mathrm{O}$ ligeramente en profundidad, no obstante estos valores no fueron 
Tabla 4. Promedios de patrones químico-estructurales obtenidos en las estratas superficiales de tres ecotipos donde se realizaron calicatas en suelos francos a franco arcilloso. Acá definimos las estrata $e 1=1-35 \mathrm{~cm} ; e 2=40-70 \mathrm{~cm} ; e 3=70-110 \mathrm{~cm}$.

\begin{tabular}{|c|c|c|c|c|c|c|c|c|c|}
\hline & \multirow[b]{2}{*}{$e 1$} & \multicolumn{2}{|c|}{$\begin{array}{l}\text { Bosque Perturbado } \\
\text { (BP) }\end{array}$} & \multicolumn{3}{|c|}{$\begin{array}{l}\text { Matorral de Transición } \\
\text { (MT) }\end{array}$} & \multicolumn{3}{|c|}{$\begin{array}{c}\text { Pradera perturbada } \\
\text { (PG) }\end{array}$} \\
\hline & & e2 & e3 & e1 & e2 & e3 & e1 & e2 & e3 \\
\hline Profundidad $(\mathrm{cm})$ & 14,50 & 17,50 & 118,25 & 14,00 & 21,50 & 65,75 & 21,25 & 48,50 & 128,8 \\
\hline Arcilla(\% & 12,25 & 11,80 & 92,00 & 27,75 & 20,50 & 31,75 & 17,20 & 19,40 & 20,33 \\
\hline Limo(\%) & 28,25 & 26,25 & 28,50 & 39,25 & 36,25 & 39,75 & 30,75 & 27,75 & 39,50 \\
\hline Arena(\%) & 56,29 & 55,14 & 56,20 & 33,00 & 43,00 & 30,00 & 52,33 & 50,33 & 43,00 \\
\hline M. Orgánica (\%) & 4,39 & 2,57 & 1,59 & 4,19 & 2,26 & 1,51 & 6,16 & 6,20 & 2,32 \\
\hline $\mathrm{pH}$ & 5,48 & 5,21 & 7,17 & 5,31 & 5,55 & 5,65 & 5,26 & 5,24 & 5,98 \\
\hline $\mathrm{CE}(\mathrm{Ms} / \mathrm{cm})$ & 0,17 & 0,10 & 0,14 & 0,14 & 0,11 & 0,08 & 0,15 & 0,13 & 0,18 \\
\hline $\mathrm{CIC}$ (meq/100gr) & 24,39 & 14,68 & 13,40 & 34,38 & 18,23 & 18,43 & 26,80 & 29,92 & 12,63 \\
\hline Nitrógeno (\%) & 0,15 & 0,07 & 0,03 & 0,17 & 0,11 & 0,08 & 0,32 & 0,57 & 0,08 \\
\hline Fosforo (mg/Kg) & 29,00 & 1,50 & 17,00 & 16,00 & 6,00 & 7,75 & 39,00 & 4,33 & 6,67 \\
\hline Potasio $(\mathrm{mg} / \mathrm{Kg})$ & 349,62 & 103,40 & 60,60 & 161,25 & 82,50 & 103,75 & 147,60 & 80,80 & 80,33 \\
\hline
\end{tabular}

significativos.

El patrón químico-nutricional, muestra que hasta una profundidad de $30 \mathrm{~cm}$, la capa orgánica superficial presenta valores en materia orgánica que bordean el $5 \%$ junto a un alto contenido de macronutrientes (Tabla 4). Los valores porcentuales de Nitrógeno presentan un alza en PG si se comparan en con ecotipo de BP o MT. A su vez es posible apreciar que la capa superficial presenta algunas deficiencias en fósforo, junto a $\mathrm{pH}$ relativamente acido (Tabla 4).

Implicancias y recomendaciones para la rehabilitación posterior a una actividad minera

El impacto provocado por las actividades de minería a cielo abierto en la región de Magallanes, lleva a la necesidad de idear planes viables que per- mitan rehabilitar exitosamente estos sitios. Es primordial contar con un modelo que integre aspectos funcionales y estructurales del ecosistema. Los resultados obtenidos en este diagnóstico mostraron algunos indicios que dan viabilidad al manejo de suelo superficial para la plantación de vegetación mediante la adecuación del modelo FRA a las condiciones de Magallanes.

Las coberturas de especies arbustivas, arbóreas y herbáceas, presentes en los diferentes ecotipos, pueden ser el resultado de perturbaciones anteriores en la zona, esto debería ser considerado al momento de establecer un plan de rehabilitación posterior a la actividad minera, dado que la presencia de especies invasivas, podrían generar efectos en el establecimiento de especies nativas. A su vez mismo es fundamental considerar los efectos facilitadores, en términos de condiciones abióticas, que 
pueden generar los micrositios en establecimiento y regeneración de especies arbóreas como Nothofagus pumilio y/o Nothofagus betuloides.

Los registros en textura y profundidad de materia vegetal presentan una variabilidad que no debería ser impedimento para desarrollar un modelo de "FRA". Este modelo debe incluir el cuidado de la cobertura superficial del suelo, en cuanto a su compactación y la combinación con los pasivos inertes.

Se pudo observar variabilidad en los patrones edáficos, pero con potencial para realizar programas de rehabilitación posterior a la extracción, teniendo en cuenta lo siguiente:

1. El sustrato con potencial de uso en las zonas de rehabilitación puede llegar a una profundidad promedio de 1.75 a 2 metros, lo que es adecuado para forestación. En los sitios registrados las texturas varían de francas a franco arenosas, con baja o nula pedregosidad, por este motivo se debe evitar los sustratos arcillosos en superficie al momento de realizar el proceso de reposición.

2. Evitar el anegamiento mediante el manejo morfológico de cobertura, imitando la poca compactación superficial propuesta por el modelo "FRA". Con esto se permitiría mayor retención hídrica y menos erosión.

En relación a la vegetación se puede recomendar lo siguiente:

1. Evaluar la factibilidad de utilizar especies arbustivas y herbáceas no competitivas dado que en sitios como Nueva Zelanda se ha observado impedimentos en colonización para Nothofagus spp. cuando hay presencia de especies invasivas.

2. ElInstitutoNacionaldeInvestigaciónAgraria (INIA) ha iniciado proyectos de investigación en especies Berberis sp y Gaulteria pumilia. Se busca el desarrollo tendiente a establecer planes de manejo y domesticación en el cultivo a través de plantaciones pilotos. Profundizar esta información nos puede llevar al uso de especies arbustivas en planes de revegetación, lo que podría ser útil cuando se busquen alternativas de establecimiento en planes de rehabilitación después de perturbaciones en sitios minados. No obstante es necesario realizar seguimientos a las asociaciones entre plántulas de bosque secundario con arbustos dado que no hay claridad en el grado facilitación o competición sitio-especifica de arbustos nativos con especies como Nothofagus pumilio.

3. Evaluar el uso de micrositios adecuados para forestación con especies arbóreas como Nothofagus pumilio, replicando las estrategias naturales encontradas en las zonas de regeneración que ocurren en los bordes de bosques o praderas, como plantación cercana a troncos caídos y arbustos nodriza.

4. Evaluar las respuestas temporales de la vegetación en las zonas estabilizadas, una vez que son removidos las estratas superficiales, mezclados y depositados.

\section{AGRADECIMIENTOS}

Los autores agradecen el apoyo en la elaboración del estudio a Mina Invierno S.A. El trabajo estuvo integrado en un proyecto $\mathrm{CORFO}$ I+D Código SC-0065 del cual la empresa es parte. Además es importante considerar el gran respaldo otorgado por la Vicerrectoría de Investigación de la Pontificia Universidad Católica de Chile.

\section{LITERATURA CITADA}

Angel, P., \& Burger J. (2002). The Appalachian Regional Reforestation Initiative and the Forestry Reclamation Approach. Published by the American Society of Mining and Reclamation (ASMR).

Ashby, W C., (1991). Surface mine tree planting in the midwest pre- and post- Public Law 95-87. In: Oaks, W., Bowden, J. (Eds.), Technologies for Success. Proceedings of the American Society for Surface Mining and Reclamation, vol. 2, Durango. American Society for Surface Mining and Reclamation, Lexington, $\mathrm{KY}$.

Burger, J., Graves D, Angel, P., Davis V. \& Zipper, C. (2005). Appalachian regional reforestation initiative. Forestry reclamation advisory no. 2. The forestry reclamation approach. Available at http://arri.osmre. 
gov/FRA/ Advisories/FRA_No.2.7-18-07. Revised.pdf Verified 11 June 2012. USDI Office of Surface Mining Reclamation and Enforcement, Washinton, DC.

Burger J., \& Zipper, C. (2011). How to Restore Forests on Surface-Mined Land. Communications and Marketing, College of Agriculture and Life Sciences, Virginia Polytechnic Institute and State University.

Camargo, L., Kossman, I., \& Imakawa, A.,(2002) Rehabilitation of Degraded Areas of Central Amazonia Using Direct Sowing of Forest Tree Seeds. Restoration Ecology Vol. 10, 636-644.

Cruz, G., \& Lara, A. (1987). Vegetación del Área de Uso Agropecuario de la XII Región, Magallanes y de la Antartica Chilena. INIA.

Dominguez, E. 2010. Aportes al conocimiento de la flora y vegetación del Monumento Natural Cueva del Milodón, Región de Magallanes (XII), Chile, Chloris Chilensis, 13(2).

Felinks, B., Pilarski, M \& Wiegleb, G. (1999). Vegetation survey in the former brown coal mining area of eastern Germany by integrating remote sensing and groundbased methods'. Applied Vegetation Science, 1, 233-240.

Gajardo, R. (1994). Vegetación Natural de Chile y distribución geográfica ( $1^{a}$ edn.). Santiago de Chile: Editorial Universitaria.

Huber, U., \& Markgraf, V. (2003) European impact on regimes and vegetation dynamics at the steppe-forest ecotone of southern Patagonia. The Holocene, (13), 567-579.

Martinic, M. (2004). La Minería del Carbón en Magallanes Entre 1868 - 2003. Historia, I(37), 129-167.

Martinic, M., Prieto, A., Arroyo, M., \& Cárdenas, R. (2011). Río Verde: Su historia y su gente (p. 151). Punta Arenas: Municipalidad de Río Verde.

Otero, L. (2006). La huella del fuego: historia de los bosques nativos, poblamiento y cambios en el paisaje del sur de Chile (p. 175). Santiago de Chile: Pehuen Editores.

Parrotta, J. \& Knowles, H. (1999) Restoration of tropical moist foresto $\mathrm{n}$ bauxite-mined lands.
Brazilian Amazon- Restoration Ecology, 7:103-116.

Promis, A., Cruz, G., Reif, A., \& Gartner, S.(2008). Bosques de Nothofagusi betuloides(Mirb.) Oerst 1871 (Fagales: Nothofagaceae) en la Patagonia Austral y Tierra del Fuego. Anales Instituto Patagonia, .36, 53-68.

Pulido, F., Díaz, B., Martínez Pastur, G. (2000). Incidencia del ramoneo del guanaco (Lama guanicoe) sobre la regeneración de lenga (Nothofagus pumilio) en bosques de Tierra del Fuego (Argentina). Invest. Agric.: Sist. Rec. For. 9, 381-394.

Puntieri, J., Raffaele, E., Martinez, P., Barthélémý, D \& Brion, C. (1999). Morphological and architectural features of young Nothofagus pumilio (Poepp. \& End.) Krasser (Fagaceae). Botanical Journal of the Linnean Society, 130, 395-410

Shulz, F., \& Wiegleb, G. (2000). Development options of natural habitats in a postmining landscape. Land Degradation \& Development, 11, 99-110.

Skousen, J., Zipper, C., Burger, J., Angel, P. Barton, C. (2011) Selecting topsoil substitutes for forestry mine soils. In R.I. Barnhisel RI (Ed.). Reclamation: sciences leading to success, Bismarck, ND (pp. 591-609). American Society of Mining and Reclamation, Lexington.

Sweigard, R., Burger, J., Zipper, C., Skousen J., Barton, C. \& Angel, P. (2007). Low compaction grading to enhance reforestation success on coal surface mines. Forestry Reclamation Advisory no. 3. Available at http://arri.osmre.gov/ FRA/Advisories/ FRA_No.3.pdf. Verified 11 June 2012. USDI Office of Surface Mining Reclamation and Enforcement, Washinton, D.C

Torbert, J., \& Burger, J. (2000). Forest land reclamation. p. 371-398. In: R. I. Barnhisel, R. G. Darmody, \& W. L. Daniels (Eds), Reclamation of Drastically Disturbed Lands. Wisconsin: Agronomy No. 41. American Society of Agronomy, Madison.

Tongway, D., \& Ludwig, J. (2011) Restoring disturbed landscapes: putting principles into practice. Island Press, Washington, DC 
Unger, P., \& Cassel, D., (1991). Tillage implements disturbance effects on soil properties related to soil and water conservation: a literature review. Soil an Tillage Research. 19, 363382.

Warhurst, A., \& Noromba, L. (2010). Corporate strategy and viable future land use: planning for closure from the outset of mining. Natural Restore Forum, 24, 153-164.

Zipper, C., Daniels, W. \& Bell, J. (1989). An experimental alternative to "approximate original contour" in steep slope terrain: environmental and policy implications. $J$. Soil Water Conservation. 44, 279-280. 
